\title{
Embedding of inkjet-printed Ag-grid/ITO hybrid transparent electrode into a plastic substrate for flexible electronic devices
}

\author{
CHANHO YANG and JIHOON KIM* \\ Division of Advanced Materials Engineering, Kongju National University, Cheonan, Chungchungnam-do 32588, Korea \\ *Author for correspondence (jihoon.kim@kongju.ac.kr)
}

MS received 16 November 2016; accepted 5 October 2017; published online 2 February 2018

\begin{abstract}
Flexible ITO/Ag-grid hybrid layers were prepared by a solution process and embedded into a plastic layer. The structural analysis confirmed that the printed layers were successfully embedded into the plastic substrate without any structural damage. The detachment of the printed ITO/Ag-grid layers from the glass substrate was carried out by dissolving a metallic sacrificial layer, which deposited on the glass substrate before the printing step. The optimum electrical and optical performance was obtained with Ag-grid pitch of $300 \mu \mathrm{m}$, leading to the sheet resistance of $2 \Omega$ per sq and the transmittance of $85 \%$.
\end{abstract}

Keywords. Solution process; inkjet; electrohydrodynamic printing; transparent electrode; flexible electrode.

\section{Introduction}

Transparent electrodes (TEs) are one of the most indispensable materials to fabricate rapidly emerging electronic devices, including flexible displays, touch panels, photovoltaic cells, light emitting diodes, thin film transistors, etc. Among various TE materials, $\mathrm{Sn}$-doped $\mathrm{In}_{2} \mathrm{O}_{3}$ (ITO) is widely used due to its excellent electrical and optical properties. However, ITO is considered unsuitable due to the following issues: (1) large fabrication cost involved in vacuum-based process and (2) lack of flexibility for future flexible electronics [1-5]. Due to these technical issues, many efforts have been devoted to preparing ITO films by solutionbased processes in a flexible platform [6-10].

The solution process based on printing technology offers an opportunity to reduce the fabrication costs from the following viewpoints: (1) efficient materials utilization due to direct deposition of the desired material at a location where it should be patterned and (2) low investment cost of the manufacturing equipment and easy scalability for large-area electronics since the printing technology does not require complicated and expensive vacuum-based equipment [11-15]. Despite these advantages of the solution process, it is reported by many research groups that the material performance of the solution-prepared ITO films needs to be further improved compared to the sputtered ITO films [16-18]. The sheet resistance of the solution-prepared ITO films is usually one-order higher than that of the sputtered films with a similar transparency. Such a high sheet resistance mainly comes from low carrier mobility [6]. Usually, the packing density of the solution-processed ITO films is not high enough compared to the sputtered films, indicating that significant amount of pores and interfaces in the solution-processed ITO films act as scattering sites for the transport of the carriers in the film.

In order to increase the conductivity of the solutionprocessed ITO films, there were several efforts to hybridize the ITO film with an assisting conduction path such as metalgrid, graphene and nanowires $[6,19,20]$.

In this paper, we present a new approach to the preparation of flexible ITO hybrid TEs. The solution-processed ITO was hybridized with a metal-grid assisting electrode patterned by electrohydrodynamic (EHD) printing. In recent times, EHD printing has gained lot of attention due to the feasibility of fine patterning with line resolution less than $10 \mu \mathrm{m}$, which is invisible to the human eyes [21-23]. The most important novelty of this work compared with other ITO-based hybrid electrodes such as ITO/graphene and Ag nanowire/ITO comes from the fabrication process itself: both ITO and Ag-grid were prepared by an inkjet printing technology. In the cases of other ITO-based hybrid electrodes, ITO is usually deposited by a vacuum-based sputtering process. These ITO-based flexible electrodes were made by transferring the assisting conducting path such as thermal-CVD-graphene and Ag nanowires onto flexible substrates and sputtering ITO over them [24,25]. Moreover, the transparency of the hybrid films is affected by the amount of the assisting conduction path introduced to the ITO film. The EHD-printed metal-grid assisting electrode in the hybrid TEs minimizes the disturbance of the transparency in the hybrid TEs. The flexibility in the hybrid TE was secured by embedding the entire hybrid TE (ITO/Ag-grid) into polydimethylsiloxane (PDMS) substrates. The electrical and optical properties of the flexible hybrid TE were investigated by modulating the line-to-line pitch in the metal grid. 
(a)

Inkjet Printing

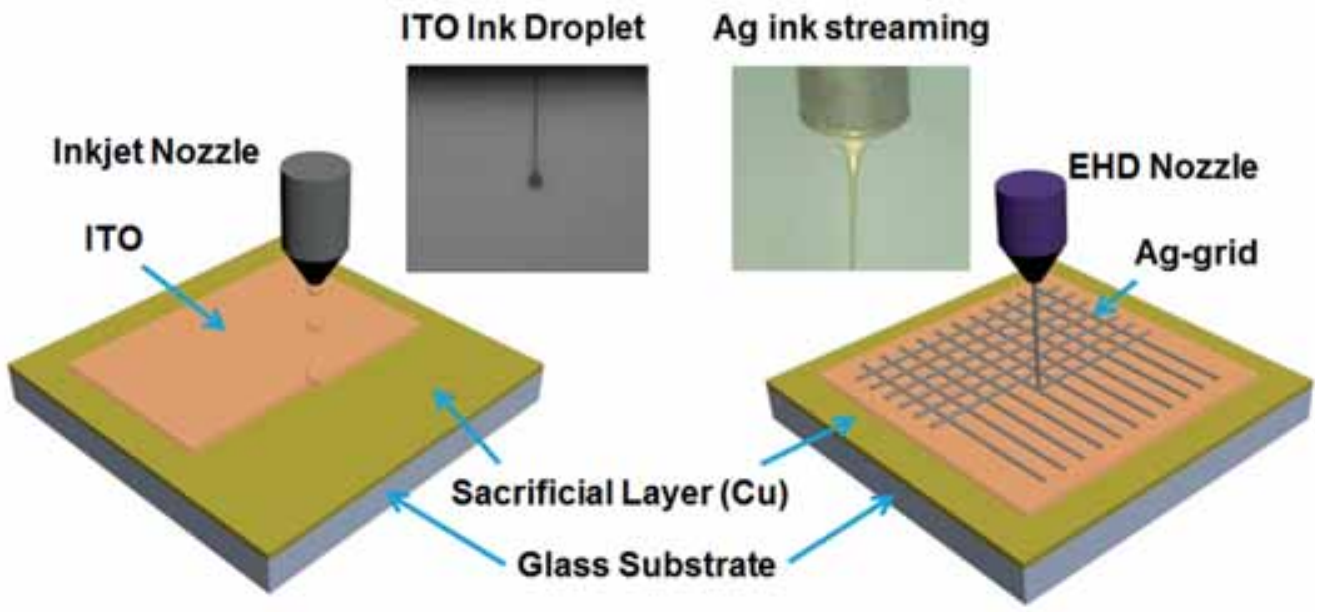

(b)

\section{PDMS Casting}

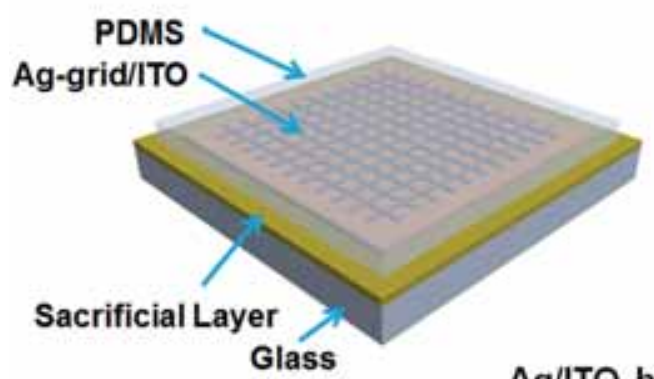

Ag/lTO hybrid TE

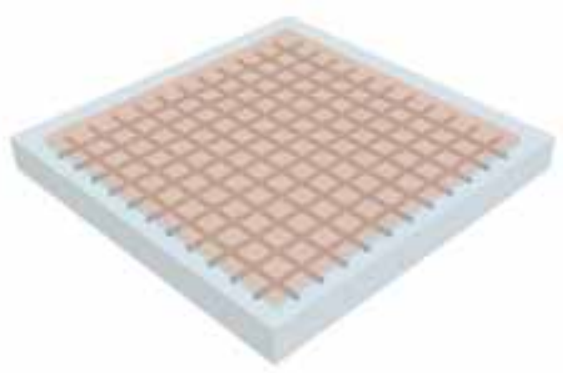

Flip over the detached PDMS layer
EHD Printing

Ag ink streaming

Detach Ag-grid//TO embedded PDMS layer
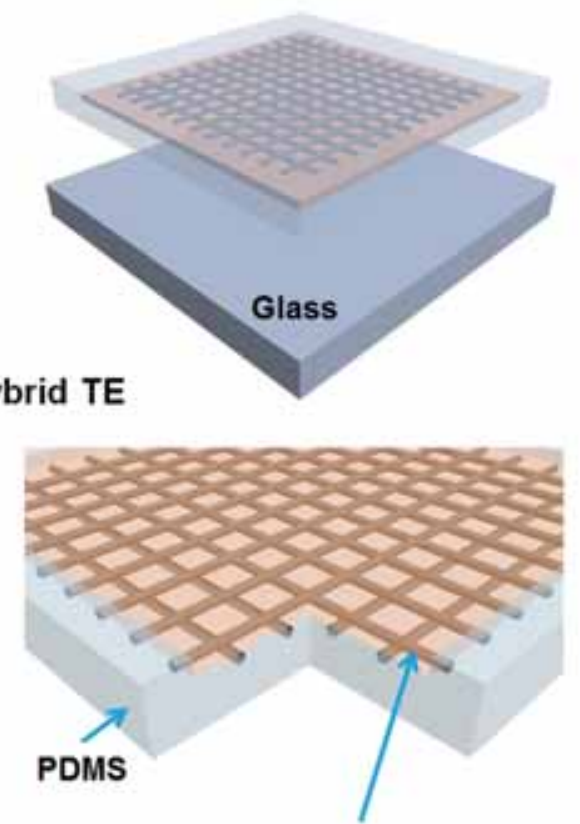

Ag-grid//TO hybrid TE embedded into PDMS

Figure 1. (a) Inkjet printing of ITO thin film and EHD printing of Ag-grid assisting electrode. (b) Fabrication process of flexible ITO/Ag-grid hybrid TE.

\section{Experimental}

Figure 1 illustrates the entire fabrication process of ITO/Ag-grid hybrid TE. The inkjet printing of ITO and EHD printing of Ag-grid assisting electrode is illustrated in figure 1a. ITO solution ink was formulated by dispersing commercial ITO nano-particles with $D_{50}=25 \mathrm{~nm}$ (Advanced Products) in N,N-dimethylformamide (DMF; boiling point: $153^{\circ} \mathrm{C}$, surface tension: $40.4 \mathrm{dyn}^{-1}$ ). ITO solid concentration in the solution was fixed at $30 \mathrm{w} \%$. The ITO ink was processed by ball-milling for $24 \mathrm{~h}$, followed by ultrasonic homogenizing process for $1 \mathrm{~h}$. An inkjet-printing unit (UJ200, UNIJET) was used to prepare the ITO films on glass substrates. The inkjet-printed ITO films were annealed at $600^{\circ} \mathrm{C}$ in vacuum by a rapid thermal annealing (RTA).

Ag-grid assisting electrode was printed on the inkjetprinted ITO films by an EHD jet printer (NP-200, ENJET) with a commercial Ag ink (NPK). The Ag ink with 50 wt\% 
solid concentration was injected into a stainless-steel nozzle with an inner diameter of $100 \mu \mathrm{m}$ by a syringe pump at the

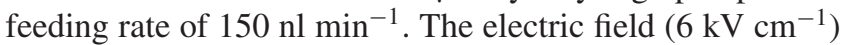
applied between the nozzle and the substrate develops a fine streaming of the Ag ink from the nozzle, which enables a fine patterning regardless of the nozzle dimension. The printing speed was fixed at $20 \mathrm{~cm} \mathrm{~s}^{-1}$.

Figure $1 \mathrm{~b}$ describes the fabrication of the flexible ITO/Aggrid hybrid TE, which consists of two processes: embedding Ag-grid/ITO hybrid TE into PDMS substrate and peeling the hybrid TE from the glass substrate.

PDMS solution was prepared by mixing PDMS base and its curing agent at a ratio of 10:1. The PDMS solution was cast over the printed Ag-grid/ITO layer on the glass substrate as described in figure $1 \mathrm{~b}$. The PDMS layer was then thermally cured at $250^{\circ} \mathrm{C}$ for $30 \mathrm{~min}$. To release the $\mathrm{Ag}$-grid/ITO hybrid TE film from the glass substrate, a metallic sacrificial layer was deposited on the glass substrate before the inkjet-printing of ITO layer. In this experiment, $\mathrm{Cu}$ layer was selected as a sacrificial layer since it can be easily deposited by a thermal evaporator and also selectively etched away with a commercially available etchant [GE103, SoonJeong]. After the PDMS curing, the Ag-grid/ITO layer was detached from the glass substrate along with the PDMS layer by dissolving the sacrificial layer in the $\mathrm{Cu}$ chemical etchant.

The electrical and optical properties of the Ag-grid/ITO hybrid TE films were characterized by Hall measurements (HMS3000, ECOPIA) and UV/visible spectrometry (V-570, JASCO), respectively. The microstructure of the Ag-grid/ITO hybrid TE was investigated using a focused-ion beam field emission scanning electron microscope (FIB FESEM, NOVA200).

\section{Results and discussion}

\subsection{Microstructure of flexible ITO/Ag-grid hybrid TE}

Figure 2 presents the microstructures of the flexible ITO/Aggrid hybrid TE embedded into PDMS substrate. The planar and tilted views of the hybrid TE are shown in figure $2 \mathrm{a}$ and b. There were no cracks observed in the inkjet-printed ITO layer. However, there is a slightly uneven surface around the Ag-grid. Figure 2c illustrates the FIB-FESEM cross-sectional image of the hybrid TE. The Ag-grid assisting electrode printed by EHD is completely embedded into PDMS and covered with the inkjet-printed ITO film (300 nm). The Ag-grid line maintained its initial printing shape without any damage even though it is embedded into the PDMS layer. The Ag line has $5 \mu \mathrm{m}$ width and $2 \mu \mathrm{m}$ height.

\subsection{Electrical property of flexible Ag-grid/ITO hybrid TE}

Figure 3 shows the electrical properties of the flexible ITO/Ag-grid hybrid TE as a function of Ag-grid pitch. The Ag-grid pitch was modulated from 500 to $100 \mu \mathrm{m}$. The sheet

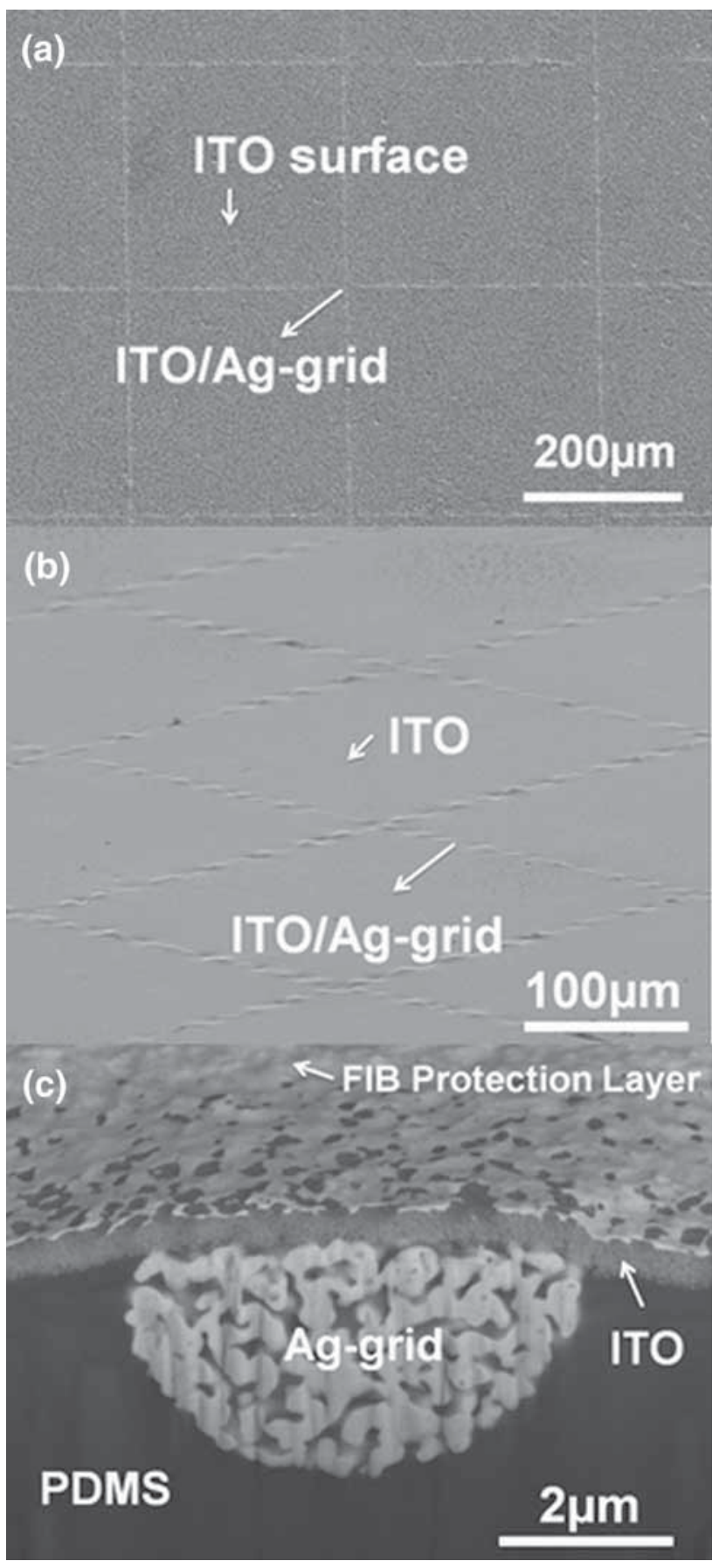

Figure 2. Microstructures of the flexible ITO/Ag-grid hybrid TE embedded into PDMS substrate: (a) planar and (b) tilted, and (c) FIB-FESEM cross-sectional views.

resistance and resistivity of the flexible ITO/Ag-grid hybrid TE decreased with decreasing Ag-grid pitch. With the Ag-grid pitch of $300 \mu \mathrm{m}$, the sheet resistance and resistivity values were $2 \Omega$ per sq and $1 \times 10^{-4} \Omega \mathrm{cm}$, respectively, which is more than 30 times smaller sheet resistance compared with the ones of the inkjet-printed ITO film without the Ag-grid assisting electrode. Table 1 presents the sheet resistance and 


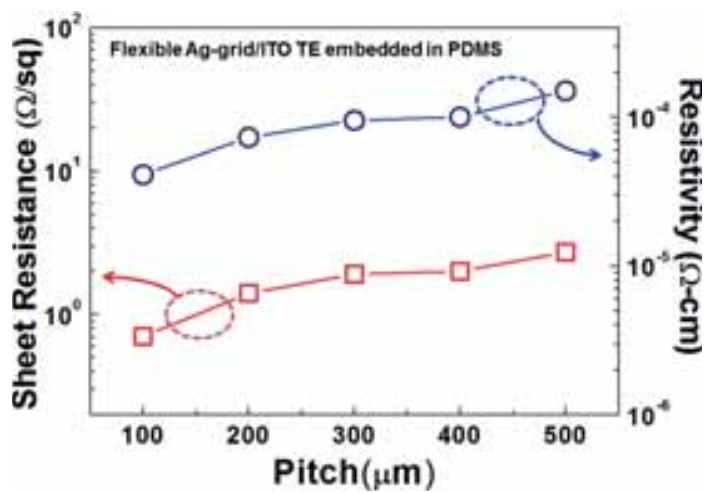

Figure 3. Electrical properties of the flexible ITO/Ag-grid hybrid $\mathrm{TE}$ as a function of Ag-grid pitch ranging from 100 to $500 \mu \mathrm{m}$.

resistivity of the inkjet-printed ITO films after annealing at $600^{\circ} \mathrm{C}$ in vacuum.

\subsection{Optical property of flexible Ag-grid/ITO hybrid TE}

Figure 4 presents the optical transmittance of the flexible ITO/Ag-grid hybrid TE at the wavelength from 300 to 800 $\mathrm{nm}$. The inset in figure 4 presents the transmittance values at $550 \mathrm{~nm}$ as a function of the Ag-grid pitch. The degradation in the transmittance can be calculated by Ag filling factor $\left(F_{\mathrm{Ag}}\right)$, which defines the area occupied by Ag-grid assisting electrode with respect to $\mathrm{Ag}$-grid free area [21]. $F_{\mathrm{Ag}}$ is obtained from the following equation:

$$
F_{\mathrm{Ag}} \approx(2 W P) / P^{2}
$$

where $W$ and $P$ are the line width and pitch of the printed line in Ag-grid, respectively. Considering the width of the EHD-printed $\mathrm{Ag}$ line $(5 \mu \mathrm{m}), F_{\mathrm{Ag}}$ becomes less than 5\% if the Ag-grid pitch becomes wider than $300 \mu \mathrm{m}$, which implies that less than $5 \%$ degradation in transmittance is expected due to the Ag-grid. Figure 4 shows that the transmittance at the Ag-grid pitch of $300 \mu \mathrm{m}$ is around $85 \%$, measured along with ITO and PMDS layers. This transmittance at the Ag-grid pitch of $300 \mu \mathrm{m}$ is in good compliance with the calculated expectation (5\% degradation in transmittance) when the ITO and PDMS layers in the hybrid TE are simultaneously taken into account.

The optical performance in the case of TE with Ag-grid assisting electrode improves at the expense of the electrical

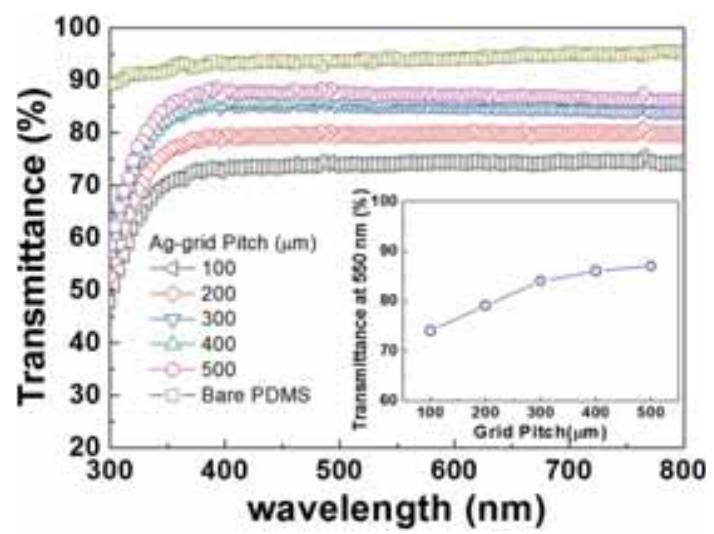

Figure 4. Optical transmittance of the flexible ITO/Ag-grid hybrid TE at wavelength from 300 to $80 \mathrm{~nm}$. The inset shows the transmittance at $550 \mathrm{~nm}$ as a function of Ag-grid pitch.

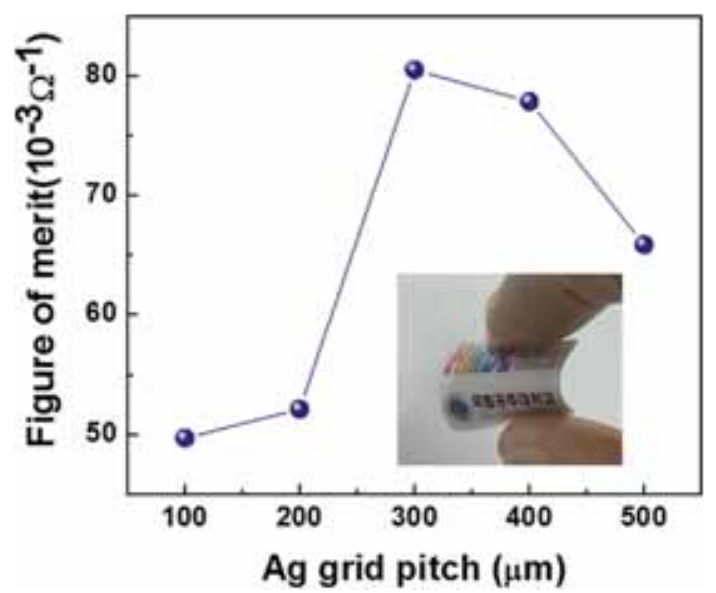

Figure 5. Figure of merit values for the flexible ITO/Ag-grid hybrid TE as a function of Ag-grid pitch. The inset shows an optical image of the flexible ITO/Ag-grid hybrid TE.

performance. There must be an optimum Ag-grid pitch for the best optical and electrical performance, which can be determined from the concept of figure of merit $\left(\phi_{\mathrm{FM}}\right)$ defined by Haacke [26]:

$$
\phi_{\mathrm{FM}}=\frac{T^{10}}{R_{\text {sheet }}}=\sigma t \exp (-10 \alpha t)
$$

Table 1. Electrical property of inkjet-printed ITO films on glass substrates after $600^{\circ} \mathrm{C}$ annealing in vacuum.

\begin{tabular}{lllc}
\hline & ITO film \#01 & ITO film \#02 & ITO film \#03 \\
\hline Sheet resistance $(\Omega$ per sq) & $3.1 \times 10^{2}$ & $2.9 \times 10^{2}$ & $2.8 \times 10^{2}$ \\
Resistivity $(\Omega \mathrm{cm})$ & $9.4 \times 10^{-3}$ & $8.9 \times 10^{-3}$ & $8.63 \times 10^{-3}$ \\
\hline
\end{tabular}


where $T, R_{\text {sheet }}, \sigma, t$ and $\alpha$ are the transmittance at a wavelength of $550 \mathrm{~nm}$, the sheet resistance ( $\Omega$ per sq), the electrical conductivity $\left(\Omega^{-1} \mathrm{~cm}^{-1}\right)$, the film thickness $(\mathrm{cm})$ and the optical absorption coefficient $\left(\mathrm{cm}^{-1}\right)$, respectively. Figure 5 shows the figure of merit variation of the flexible ITO/Ag-grid hybrid TE with the Ag-grid pitch. The figure of merit increases with increasing Ag-grid pitch up to $300 \mu \mathrm{m}$ since the transmittance values are predominant in the equation compared with the sheet resistance values. The maximum $\phi_{6}$ value $\left(82 \times 10^{-3} \Omega^{-1}\right)$ reached at the Ag-grid pitch of $300 \mu \mathrm{m}$. Further increase in the Ag-grid pitch beyond $300 \mu \mathrm{m}$ resulted in the decrease of $\phi_{\mathrm{FM}}$.

\section{Conclusions}

In this study, ITO/Ag-grid hybrid layers were embedded into a plastic layer (PDMS in this study) and used as a flexible TE. Both ITO and Ag-grid assisting electrode were prepared by the inkjet printing and EHD printing, respectively. $\mathrm{A} \mathrm{Cu}$ sacrificial layer was introduced to the glass substrate and used to detach the printing layers (Ag-grid/ITO) from the substrate. The sheet resistance of the flexible ITO/Ag-grid hybrid TE is smaller than $2 \Omega$ per sq and decreases with decreasing Ag-grid pitch. The optical transmittance remains greater than $85 \%$ for the Ag-grid pitch of $300 \mu \mathrm{m}$ or greater. The $\phi_{\mathrm{FM}}$ increases with increasing Ag-grid pitch up to $300 \mu \mathrm{m}$. Further increase in the Ag-grid pitch led to the decrease of $\phi_{\mathrm{FM}}$.

\section{Acknowledgements}

This work was supported by 2015 Internal Research Program funded by Kongju National University.

\section{References}

[1] Krebs F C, Jorgensen M, Norrman K, Hagenmann O, Alstrup J, Nielsen T D et al 2009 Sol. Energy Mater. Sol. Cells 93 422
[2] Krebs F C 2009 Sol. Energy Mater. Sol. Cells 93394

[3] Spanggaard H and Krebs F C 2004 Sol. Energy Mater. Sol. Cells $\mathbf{8 3} 123$

[4] Krebs F C 2009 Sol. Energy Mater. Sol. Cells 931636

[5] Krebs F C 2009 Sol. Energy Mater. Sol. Cells 93465

[6] Hwang M S, Jeong B Y, Moon J, Chun S K and Kim J 2011 Mater. Sci. Eng. B $\mathbf{1 7 6} 1128$

[7] Koo B-R and Ahn H-J 2016 Ceram. Int. 42509

[8] Jeong J-A, Lee J, Kim H, Kim H-H and Na S-I 2010 Sol. Energy Mater. Sol. Cells 941840

[9] Korosi L, Papp S and Dekany I 2011 Thin Solid Films 5193113

[10] Sunde T O L, Garskaite E, Otter B, Fossheim H E, Saeterili R, Holmestad R et al 2011 J. Mater. Chem. 2215740

[11] Sirringhaus H, Kawase T, Friend R H, Shimoda T, Inbasekaran M, Wu W et al 2000 Science 2902123

[12] Stree R A, Wong W S, Ready S E, Chabinyc M L, Arias A C, Limb S et al 2006 Mater. Today 932

[13] Kawase T, Sirringhaus H, Friend R H and Shimoda T 2001 Adv. Mater. 131601

[14] Bharathan J and Yang Y 1998 Appl. Phys. Lett. 722660

[15] Hebner T R, Wu C C, Marcy D, Lu M H and Sturm J C 1998 Appl. Phys. Lett. 72519

[16] Duta M, Anastasescu M, Calderon-Moreno J M, Predoana L, Preda S, Nicolescu M et al 2016 J. Mater. Sci.: Mater. Electron. 274913

[17] Muramatsu A, Kanie K, Sasaki T and Nakaya M 2016 KONA Powder Part. J. 33340

[18] Shin H-I, Kim K-H, Kim T-W and Kim H-K 2016 Ceram. Int. $\mathbf{4 2} 13983$

[19] Jung S, Lee S, Song M, Kim D-G, You D S, Kim J-K et al 2014 Adv. Energy Mater. 41300474

[20] Lim J-W, Cho D-Y, Kim J, Na S-I and Kim H-K 2012 Sol. Energy Mater. Sol. C 107348

[21] Jang Y, Kim J and Byun D 2013 J. Phys. D Appl. Phys. 46 155103

[22] Vishwanath S K, Kim D G and Kim J 2014 Jpn. J. Appl. Phys. 53 05HB 11

[23] Lee Y, Jin W-Y, Cho K Y, Kang J-W and Kim J 2016 J. Mater. Chem. C 47577

[24] Liu J, Yi Y, Zhou Y and Cai H 2016 Nanoscale Res. Lett. 11 108

[25] Seo H, Yun H D, Kwon S-Y and Bang I C 2016 Nano Lett. 16 932

[26] Haacke G 1976 J. Appl. Phys. 474086 